\title{
X-RAY OBSERVATIONS OF THE GALACTIC CENTRE
}

\author{
G. K. SKINNER \\ Dept. of Space Research \\ University of Birmingham \\ Birmingham, B15 2TT \\ England
}

\begin{abstract}
Observations of the galactic centre region in the photon energy range 2-500 keV are reviewed. Point sources, transients, bursts and a patch of apparently diffuse emission $\sim 1^{\circ}$ in extent have all been observed. The relatively detailed information obtained with the Einstein observatory just above the bottom edge of the $x$-ray window is starting to be supplemented by observations at higher photon energies. Although there is known to be a strong, variable, source of high energy $x$-rays somewhere in the region there is little reason to associate it with Sgr A West, which is detectable, but relatively weak, in the energy range below $30 \mathrm{keV}$ where detailed measurements have been possible.
\end{abstract}

\section{INTRODUCTION}

The very high column densities of interstellar material in the direction of the galactic nucleus block ionizing radiation with photon energies less than $\sim 1-2 \mathrm{keV}$. This review will consider what we know of the galactic centre regions from observations between this limit and the $511 \mathrm{keV}$ annihilation line, where we may assume the gamma ray domain to start. Compared with other wavelengths, the angular resolutions which have been obtained with $x$-rays and gamma-rays are poor, particularly at higher energies. Emission described as 'consistent with the galactic centre' may in many cases have originated from projected distances of $\sim 1 \mathrm{kpc}$ from the nucleus. Only at low energies is it possible to be at all specific about source locations and in particular about the central $<0.5^{\circ}$. As, moreover, the situation at higher energies has changed little since the review of Matteson (1982), the majority of this review will be concerned with photon energies below $30 \mathrm{keV}$; higher energies will be considered only in $\S 6$. All luminosities quoted are referred to a galactic centre distance of $10 \mathrm{kpc}$.

\section{OBSERVATIONS IN THE CONVENTIONAL X-RAY BAND (1-10 keV)}

$\mathrm{X}$-ray emission from the region of $\mathrm{Sgr} \mathrm{A}$ was observed with some of the very earliest rocket and balloon instruments but detailed observations started with the Uhuru satellite in 1971 which detected X-rays from a region about $2^{\circ}$ in extent (Fig.1) centred on Sgr A (Kellogg et al., 1971). With an angular resolution of $0.5 \times 5^{\circ}$, the observations were consistent with either an extended source or a complex of at least three sources; it is now clear that in fact both are present. 
Fig.1 Polygon: The maximum extent of the galactic centre $x$-ray emission observed with Uhuru (Kellogg et al., 1971). The data were also consistent with a line of emission or at least three discrete sources. Circles: positions of three x-ray burst sources (Lewin et al., 1976).

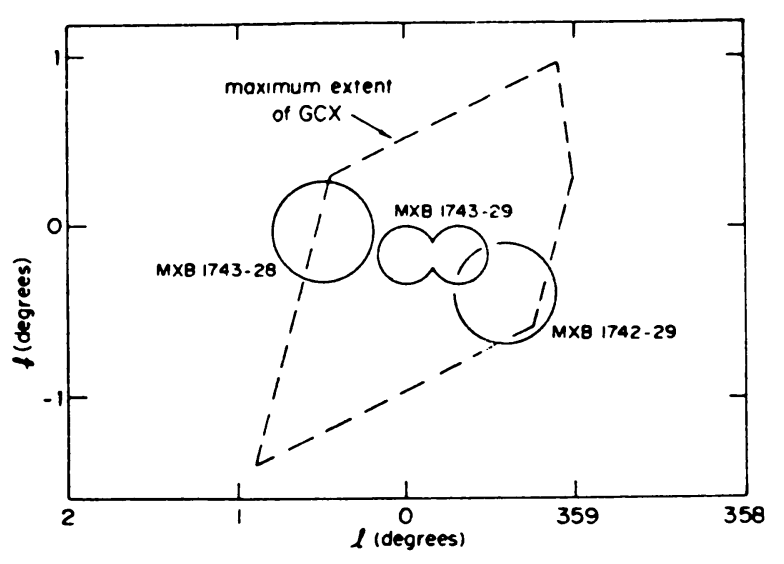

Subsequent satellite (Jernigan et al., 1978; Skinner, 1979) and rocket (Cruddace et al., 1978; Proctor et al., 1978) experiments with somewhat better angular resolution established the presence of a number of point sources within the Uhuru source region. One source, A1742-294, appears to be variable, but persistent, and is typically the brightest source in the region at these energies. A1742-294 must have been at a lower than average state at the time of the Uhuru observations, otherwise it alone would have accounted for all of the flux seen. It lies $30^{\prime}$ from $\mathrm{Sgr} \mathrm{A}^{*}$ and there is little to distinguish it from the $\sim 8$ 'galactic bulge sources' which are clustered in the central $\pm 20^{\circ}$ of the galactic plane and which are reasonably well understood in terms of models in which a neutron star accretes from a low mass binary companion (see review by McClintock and Rappaport, 1985).

\subsection{TIME VARIABILITY - TRANSIENTS AND BURSTS}

Several point sources have been detected, and sometimes confirmed in more than one observation, but then found not to have persisted for more than $\sim$ months (e.g., GX+0.2-0.2, Cruddace et al., 1978; Skinner, 1979). The ubiquity of $x$-ray transients, mostly brightening rapidly and then dying away over a period of months, established by the Uhuru, Ariel-5 and $S A S-3$ generation of satellite experiments, makes it likely that at least some of the highly variable sources are transients occurring in the high density of stars in the central few hundred parsecs.

GX359+2 (=4U1735-28), which was seen to brighten to $\sim 500 \mu \mathrm{Jy}$ and later disappear during the Uhuru observations (Kellogg et al., 1971), was probably an example of such a source. More information is available in the case of A1742-28 (Eyles et al., 1975; Branduardi et al., 1976), a transient which interestingly lay very close to the direction of Sgr A. It was seen during Ariel-5 observations of the region in 1975 and reached a peak intensity more than twice that of the Crab Nebula. Although the original 2.4' square error box included Sgr $\mathrm{A}^{*}$, further analysis of the data led to a reduction in the uncertainties (Wilson et al., 1977) and located the source $54 \pm 27^{\prime \prime}(90 \%$ confidence limits) SW of $\mathrm{Sgr} \mathrm{A}^{*}$. The refined position is still consistent with that of a radio transient seen during the $x$-ray outburst by Davies et al. (1976). If the further identification with a $\mathrm{K}$ dwarf coincident with the radio source is also correct, as suggested by Murdin et al. (1980), then the distance is only $\sim 1.2 \mathrm{kpc}$ and the near alignment with the galactic centre is fortuitous. The very strong low energy cutoff is variable (Branduardi et al., 1976), so it must be due largely to absorption by material 
local to the source and cannot be taken as evidence for a source position close to the nucleus.

In the galactic centre region there is known to be a number of sources of $x$-ray bursts (events lasting $\sim 10 \mathrm{~s}$ - see review by Lewin and Joss, 1981), but their locations are rather uncertain. The most detailed study remains that of Lewin et al. (1976) who observed the region with ANS for a total of about 200 hours. 28 bursts were seen from at least three different sources but it was only possible to locate the sources to about $0.3^{\circ}$. Bursts were also seen, but could not be localised, with the Einstein MPC (two possible bursts in $7.6 \mathrm{~h}$ - Watson et al., 1981), Hakucho (12 bursts - Inoue et al., 1981; Hayakawa, 1981) and Exosat (Turner, private communication, quoted by Warwick et al., 1988). A burst observed during the 7 hours of Spacelab-2 galactic centre observations has been shown to originate from the persistent point source, SLX1744-299 (Skinner et al., in preparation). There has been no confirmation of the frequent very weak x-ray bursts reported by Proctor et al. (1978) on the basis of their 6 minute rocket observation.

$\mathrm{X}$-ray bursts are thought to arise from the sudden nuclear burning on the surface of a neutron star of an accumulation of matter accreted from a binary companion. Elsewhere in the galactic plane they seem to be associated with low-mass $x$-ray binaries in which the steady luminosity is not too high. As with transients, the incidence of $x$-ray burst sources and of $x$-ray bursts in the central $100-200 \mathrm{pc}$ is qualitatively consistent with an enhanced space density of Pop II stars.

It has been found that during the evolution of many $x$-ray bursts the radius of the emitting sphere implied by the combination of luminosity and temperature often remains constant at a value consistent with that expected for a neutron star. Corrections are necessary for the structure of the envelope, in which Compton scattering dominates so that the black-body approximation is not valid and the colour temperature is not the same as the effective temperature (London, Taam and Howard, 1984). To the extent that these effects and the equation of state of the neutron star are understood, an estimate of the distance of the burst source is possible (Ebisuzaki, Hanawa and Sugimoto, 1984; Foster, in preparation). Although subject to large uncertainties, such estimates (and ones obtained by a related approach comparing the peak burst luminosity with the Eddington limit, e.g., Tanaka, 1987) are interesting as they are entirely independent of other distance measures. Distances to the galactic centre somewhat less than the currently adopted $8.5 \mathrm{kpc}$ tend to be favoured by such calculations.

\subsection{HIGHER RESOLUTION MEASUREMENTS}

The first high-sensitivity $\mathrm{x}$-ray images of the galactic centre were obtained by Watson et al. (1981) with the Einstein Observatory. Fig. 2 shows schematically the image obtained with the IPC detector at the focal plane, giving an angular resolution $\sim 1^{\prime}$ over a $1^{\circ}$ field. Except for the persistent source A1742-294, mentioned above, which is at the edge of the field of view, the next strongest discrete source is \#3, which is almost certainly to be identified with Sgr A West. The compact component Sgr A* lies only $20^{\prime \prime}$ from the centre of the $\mathbf{1}^{\prime}$ error circle. The most remarkable feature of the Einstein IPC images, though, is an apparently diffuse component, $\sim 33^{\prime} \times 18^{\prime}(\Delta l \times \Delta b)$ in maximum extent, responsible for $\sim 85 \%$ of the emission from the central region (i.e., excluding A1742-294). 
Fig.2 Schematic map of the Einstein Observatory images of the galactic centre (Watson et al., 1981). Numbered circles represent discrete sources with approximate $90 \%$ error radii. Contours (broken where uncertain) indicate the diffuse emission.

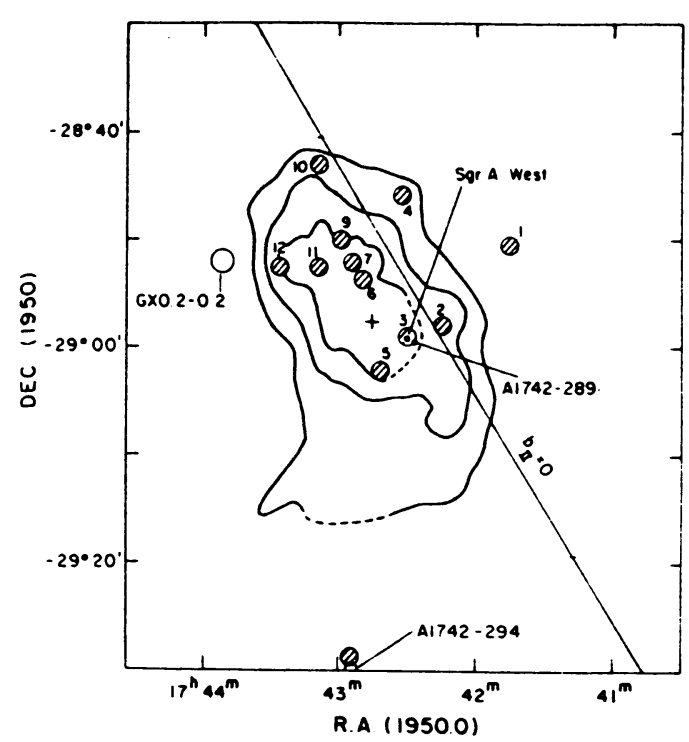

The combination of the absorption at low energies due to interstellar matter (see $\$ 5$ below) and the upper energy limit of the Einstein instrument at $\sim 5 \mathrm{keV}$ mean that the observations of Watson et al. were effectively constrained to a band of photon energies between 2 and $4.5 \mathrm{keV}$ (Fig.3). More recently, two instruments have obtained images at higher energies, although with somewhat poorer sensitivity and resolution.

The first of these was the Spartan-1 payload (Kawai et al., 1988), which scanned the area in June 1985 with collimated proportional counters $\left(5^{\prime} \times 3^{\circ}\right)$ in six directions,

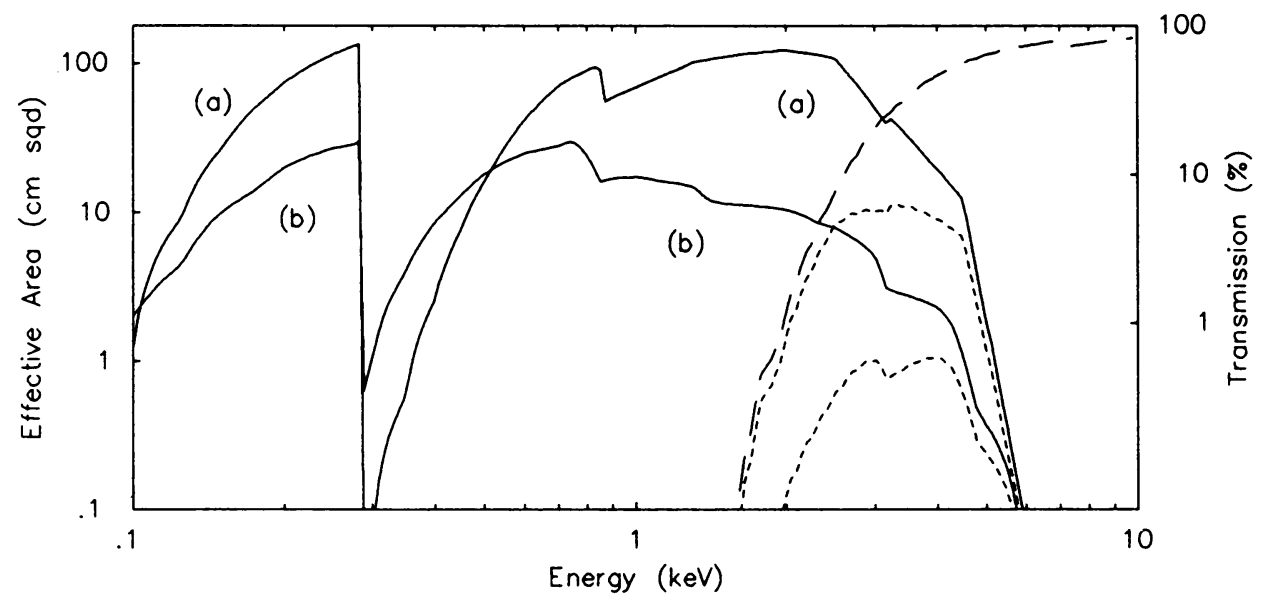

Fig.3 The effective area of the Einstein observatory telescope with (a) the IPC and (b) the $H R I$ in the focal plane. The dashed line shows the transmission of the interstellar medium for $N_{H}=10^{23} \mathrm{~cm}^{-2}$. The dotted lines show the effective areas when looking through such a column. 
allowing a MEM (maximum entropy method) image to be reconstructed. The instrument operated in the energy range 2-10 keV. Shortly after the Spartan-1 flight, the Spacelab-2 mission carried a pair of coded-mask telescopes which imaged the galactic centre region with $3^{\prime} \times 3^{\prime}$ and $12^{\prime} \times 12^{\prime}$ resolution and extended the energy range up to $30 \mathrm{keV}$ (Skinner et al., 1987).

The general form of the Spartan-1 and Spacelab-2 images are very similar (Figs. 4,5). Both show the same pattern of 4 bright sources in the central group and a pool of extended emission, centred approximately on Sgr A* $^{*}$ These observations were separated by only six weeks, but there is independent evidence that at least 3 of these sources are long-term features. These are (a) A1742-294 (see above), (b) 1E1743.1-2843, one of the point sources seen in the Einstein galactic centre observations of Watson et al. 6 years earlier, (c) 1E1740.7-2942 - which was observed in the Einstein galactic plane survey (Hertz and Grindlay, 1984). The fourth strong source (referred to as SLX1744-299 or SP1744.2-2959) is the one from which the $x$-ray burst in the Spacelab-2 data (mentioned in \$2.1) was observed. It lies $3^{\prime}$ from the remarkable non-thermal radio source G359.305-0.84 (Yusef-Zadeh and Bally, 1987), but both the Spartan-1 and Spacelab-2 error boxes exclude with high significance the possibility that the two are associated.

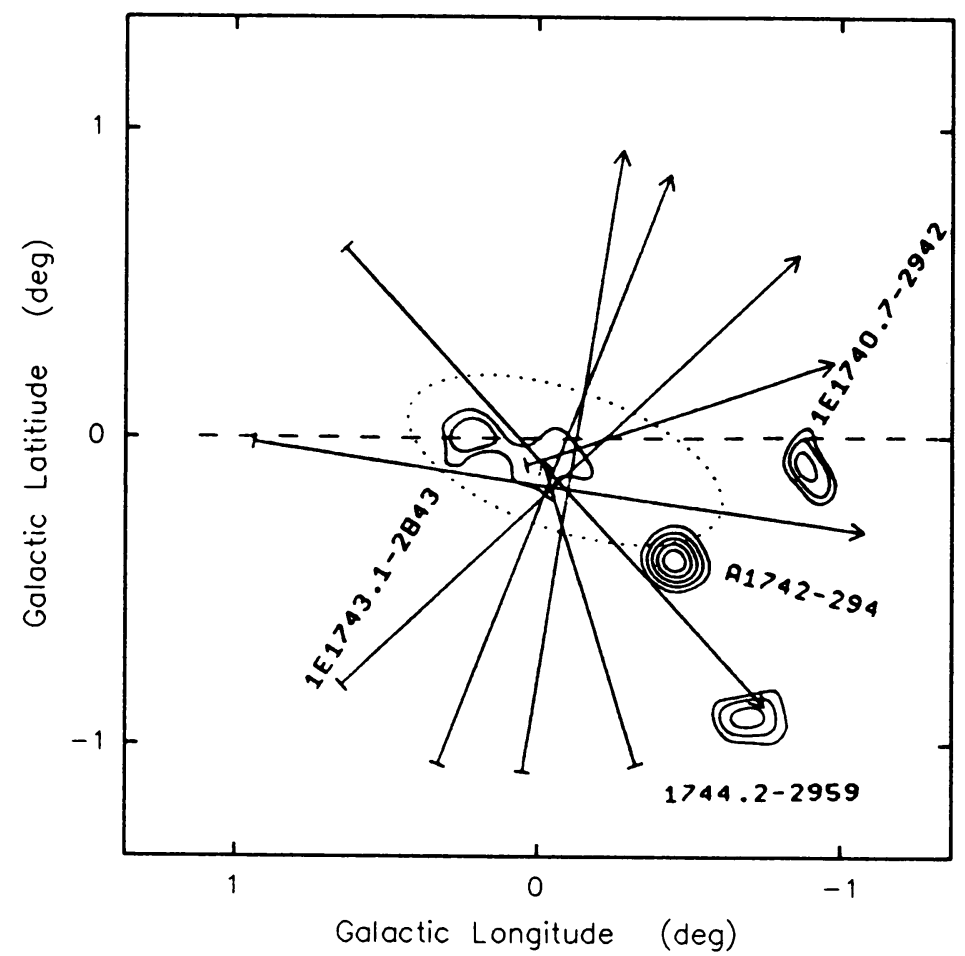

Fig.4 Maximum entropy method reconstruction of the 2-10 keV x-ray brightness of the galactic centre region from Spartan-1 data (Kawai et al., 1988). Energy band 2-10 keV. Arrows indicate the scan directions of the slit collimator. The dashed ellipse shows the extent of the centrally localised diffuse source, as determined by fitting. Contours spaced by ratio of $e$. 

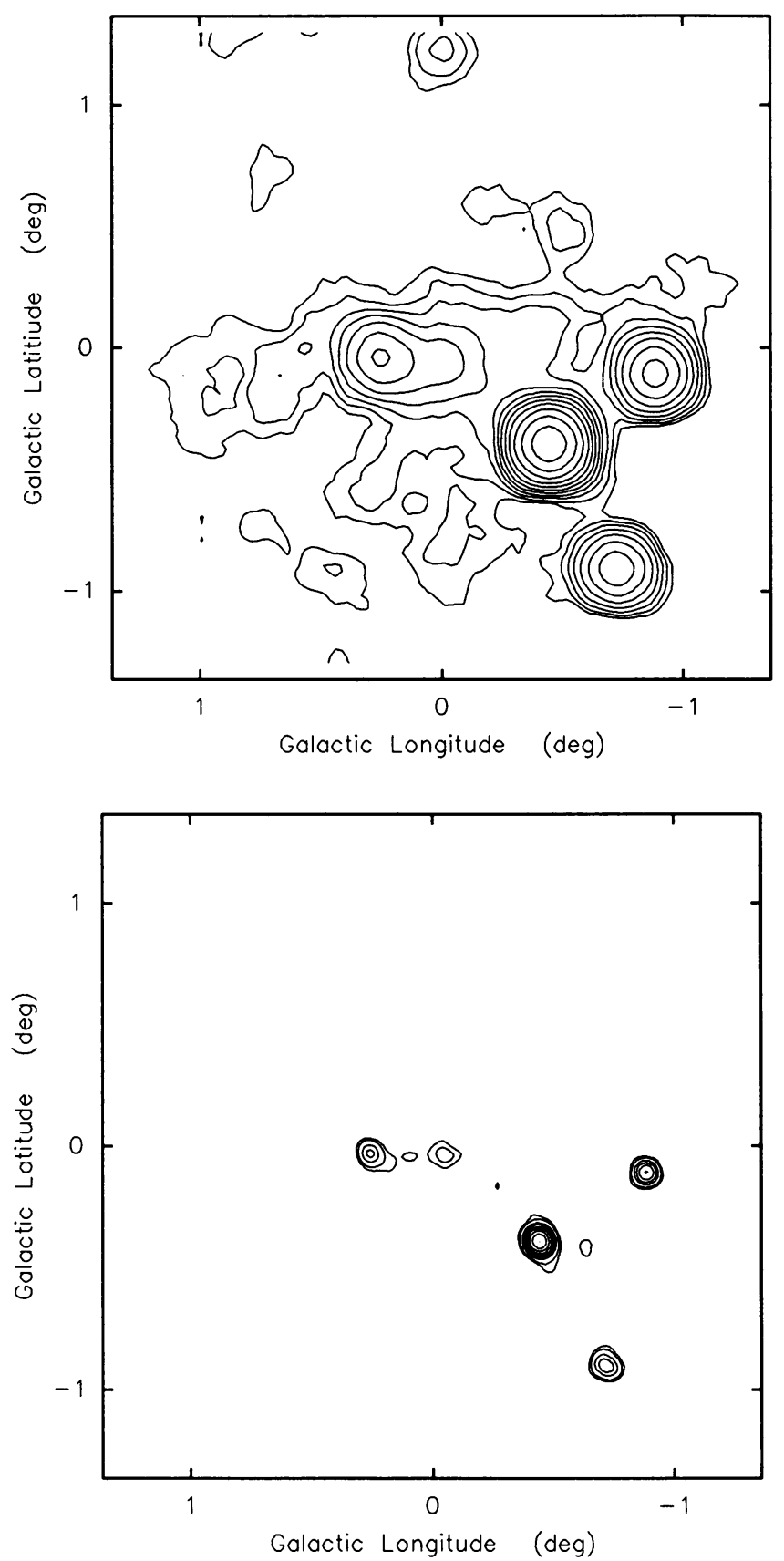

Fig.5 (a) Image obtained with the 12 arcminute resolution Spacelab-2 telescope. Energy band $3-32 \mathrm{keV}$. Contours spaced by ratio of 1.5. (b) The corresponding image from the 3 arcminute resolution telescope. The weak central peak corresponds to Sgr $A^{*}$ within the precision of the measurement. 
The situation in the crucial region immediately around $\operatorname{Sgr} \mathrm{A}^{*}$ is less clear cut and will be discussed in $\S 4$ below.

\section{DIFFUSE EMISSION}

The surface brightness and spectrum of the diffuse region found from the Einstein observations by Watson et al. (1981) corresponds to about $10^{-10} \mathrm{erg} \mathrm{cm}^{-2} \mathrm{~s}^{-1} \mathrm{sr}^{-1}(2-10$ $\mathrm{keV}$ ), over a mean radius of 0.2 degrees. Analyses of the Spartan-1 and Spacelab-2 observations yield similar figures for the central region (within about $20 \%$, after allowing for the different spectral ranges). However both of these instruments indicate that the extent of the diffuse emission is several times larger than the $\sim 3^{\prime} \times$ $18^{\prime}$ region apparent in the Einstein data, leading to luminosities 2-4 times larger (4-8.10 $\left.0^{36} \mathrm{erg} \mathrm{s}^{-1}\right)$. This is probably due to a combination of the limited field of view of the Einstein telescope and the emission being more extended at high energies.

A uniform diffuse emission across the entire region surveyed was also seen in the Spartan-1 data. This was of similar brightness to the central part of the galactic ridge seen with Exosat by Warwick et al. (1985) to extend $\sim 80^{\circ}$ along the galactic plane, and so is probably not directly associated with the galactic centre. The operation of the Spacelab-2 coded-mask instrument was such that a uniform level would not necessarily be seen.

It is not clear if either the galactic ridge or the emission around Sgr A West are truly diffuse or whether they are simply the effect of a large number of unresolved sources. The tightest constraints come from the Einstein galactic centre observations, where Watson et al. argue from the smoothness of the emission that $\gtrsim 3000$ sources, of mean luminosity $\lesssim 10^{33} \mathrm{erg} \mathrm{s}^{-1}$, would have to be responsible for the $33^{\prime} \times 18^{\prime}$ component which they observe. (Similarly, Warwick et al. conclude that if individual discrete objects are responsible for the ridge observed by Exosat they would have been observed in the Einstein galactic plane survey of Hertz and Grindlay (1984) if they were more luminous than $10^{33.5} \mathrm{erg} \mathrm{s}^{-1}$; see also Worrall and Marshall, 1983.) There are no clear candidates for a class of low luminosity sources capable of providing the observed flux, the emission from most isolated stars being too soft.

One mechanism by which truly diffuse emission could arise would be inverse Compton scattering of a population of low energy photons by relativistic electrons. The greatest photon energy density is likely to be in far-infrared photons, for which the electron energy would need to be $E_{e}$, where

$$
\left(\frac{E_{e}}{100 \mathrm{MeV}}\right)=3.2\left(\frac{E_{x}}{5 \mathrm{keV}}\right)^{1 / 2}\left(\frac{\lambda}{100 \mu m}\right)^{1 / 2}
$$

The synchrotron radiation to which such electrons would give rise in $\mu$ Gauss magnetic fields would be at relatively low frequencies

$$
\left(\frac{\nu}{100 M H z}\right) \approx\left(\frac{E_{x}}{5 k e V}\right)\left(\frac{B}{100 \mu G a u s s}\right)\left(\frac{\lambda}{100 \mu m}\right)
$$

and so such a population cannot be excluded; for consistency with the observed $x$-ray spectrum (photon index, $m \sim 2$ ) a rather steep electron energy spectrum (energy index, $n=2 m-1 \sim 3$ ) would be needed. 
Synchrotron radiation from much higher energy electrons,

$$
\left(\frac{E_{e}}{10^{7} \mathrm{MeV}}\right)=2.7\left(\frac{E_{x}}{5 \mathrm{keV}}\right)^{1 / 2}\left(\frac{B}{100 \mu \text { Gauss }}\right)^{-1 / 2}
$$

could also be responsible. The number of electrons needed is not totally unreasonable in the case of the galactic ridge emission, which is an order of magnitude more luminous, Bhat, Kifune and Wolfendale (1985) have argued that the required electrons could be produced by $\sim 30 \mathrm{Cyg}$ X3 -like sources.

A third possible origin for the diffuse flux is suggested by the observations of Koyama et al. (1986) who detected diffuse flux from the galactic plane $\left(280^{\circ}<l<340^{\circ}\right)$ with a spectrum containing a pronounced $\mathrm{Fe} \mathrm{K}$-fluorescence line at $6.7 \mathrm{keV}$, indicating thermal bremsstrahlung from a plasma with $T \sim 10^{7}-10^{8} K$. The spectrum of the galactic centre diffuse emission indicates $T \sim 10^{8} \mathrm{~K}$, within about a factor of 2 , requiring an emission measure $\left(\int n_{i} n_{e} d V\right)$ of $(2 \pm 1) \times 10^{59} \mathrm{~cm}^{-3}$ to produce the luminosity observed inside a radius of $40 \mathrm{pc}$. The implied density $\left(n_{e} \gtrsim 0.1-0.2 \mathrm{~cm}^{-3}\right)$ and pressure $\left(\sim 10^{7} \mathrm{~cm}^{-3} \mathrm{~K}\right)$ are rather difficult to reconcile with other known components. Lastly, Compton scattering of photons from discrete sources will contribute to the diffuse flux ( $\$ 5 \mathrm{c}$ below) and three photon decay of positronium (Leventhal, MacCallum and Stang, 1978) will give rise to a continuum with a spectrum rising towards $511 \mathrm{keV}$ and could be responsible for a significant diffuse component at energies $\gtrsim 100 \mathrm{keV}$.

\section{SGR A}

The source corresponding to Sgr A West is indistinguishable from a point source in the Einstein IPC data (i.e., at $\mathbf{1}^{\prime}$ resolution). From the fact that the nuclear source was not seen with the HRI detector (capable of $2^{\prime \prime}$ resolution), in the Einstein telescope focal plane there is a hint that the source may be extended on the sub-arcminute scale. As, however, the sensitivity of the HRI in the relevant energy band is much lower than that of the IPC

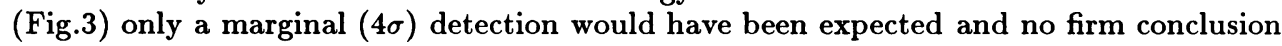
can be reached.

Thus the detected flux could be associated with the extended component of Sgr A West, with $\mathrm{Sgr} \mathrm{A}^{*}$, or could be simply the integrated emission from a very high stellar density. Estimates of the intrinsic luminosity are critically dependent on the spectrum and absorbing column density assumed, but using $N_{H}=6 \times 10^{22} \mathrm{~cm}^{-2}$, Watson et al. (1981) find $1.5 \times 10^{35} \mathrm{erg} \mathrm{s}^{-1}$ for the $0.5-4.5 \mathrm{keV}$ luminosity of this component for a spectrum with $k T=5 \mathrm{keV}$.

Higher energy observations are crucial (for example stellar emission from $10^{7}$ dwarfs would be expected to have $k T<1 \mathrm{keV}$ ) but are at first sight conflicting. Kawai et al. (1988) find no evidence in the Spartan-1 data for any x-ray emission from Sgr A West and conclude that it must have been at least a factor of four weaker than during the Einstein observations. Spacelab-2 data indicate a flux 7 times greater than would have been expected based on the Einstein observations (Skinner et al., 1987).

These results should not necessarily be taken to imply strong variability during the 6 week interval between these measurements. The Spartan-1 upper limit $\left(2\right.$ counts $\left.\mathrm{s}^{-1}\right)$, corresponds to a flux about equal to that detected by Spacelab- $2\left(5.2 \times 10^{-11} \mathrm{erg} \mathrm{cm}^{-2} \mathrm{~s}^{-1}\right.$, 3-30 keV). The conclusion that the source was weaker than during the Einstein observations only arises if 1E1743.1-2843 is used as a comparison. In fact, due either to variability or to spectral effects, 1E1743.1-2843 was $\sim 50$ times brighter during the 


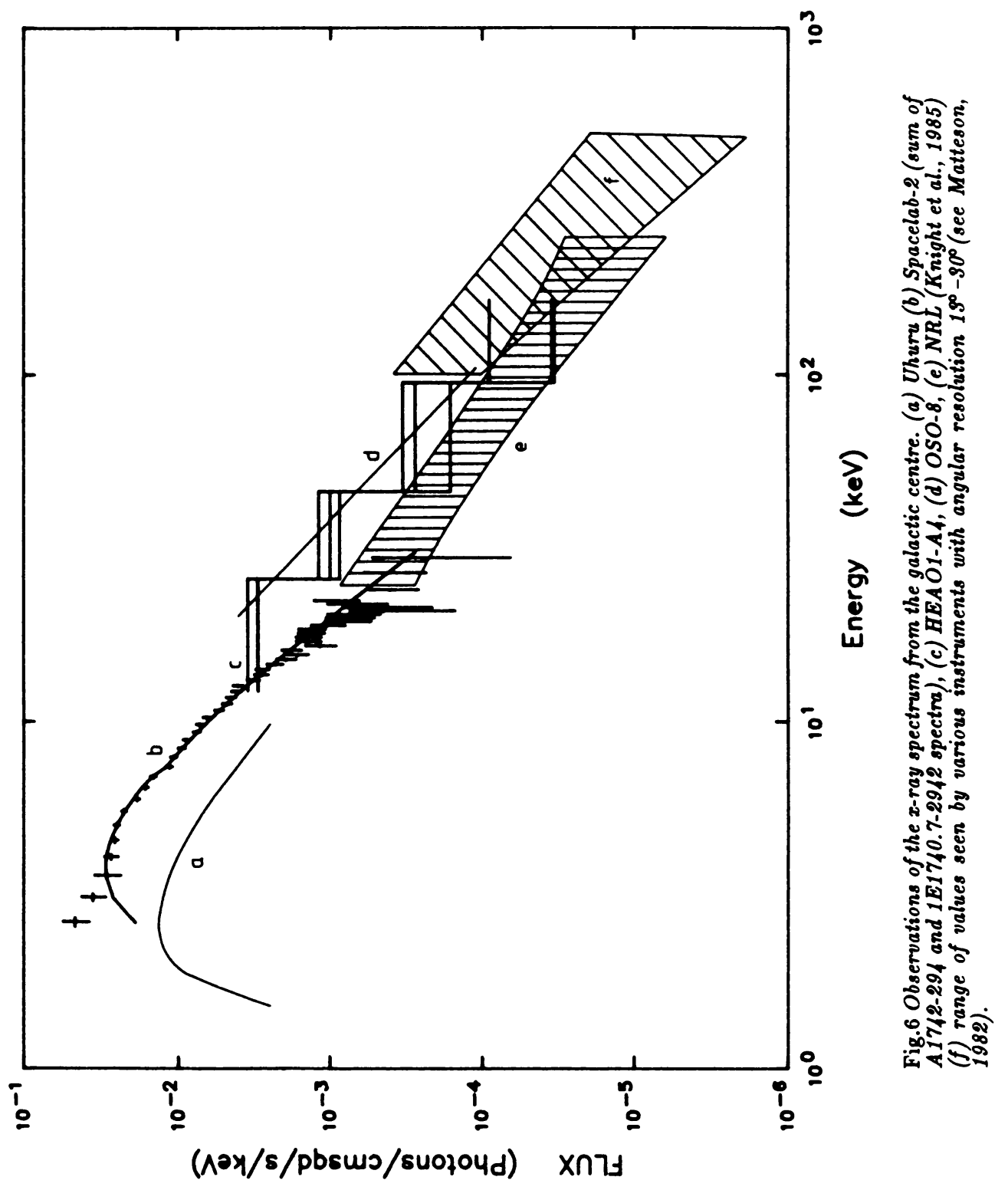




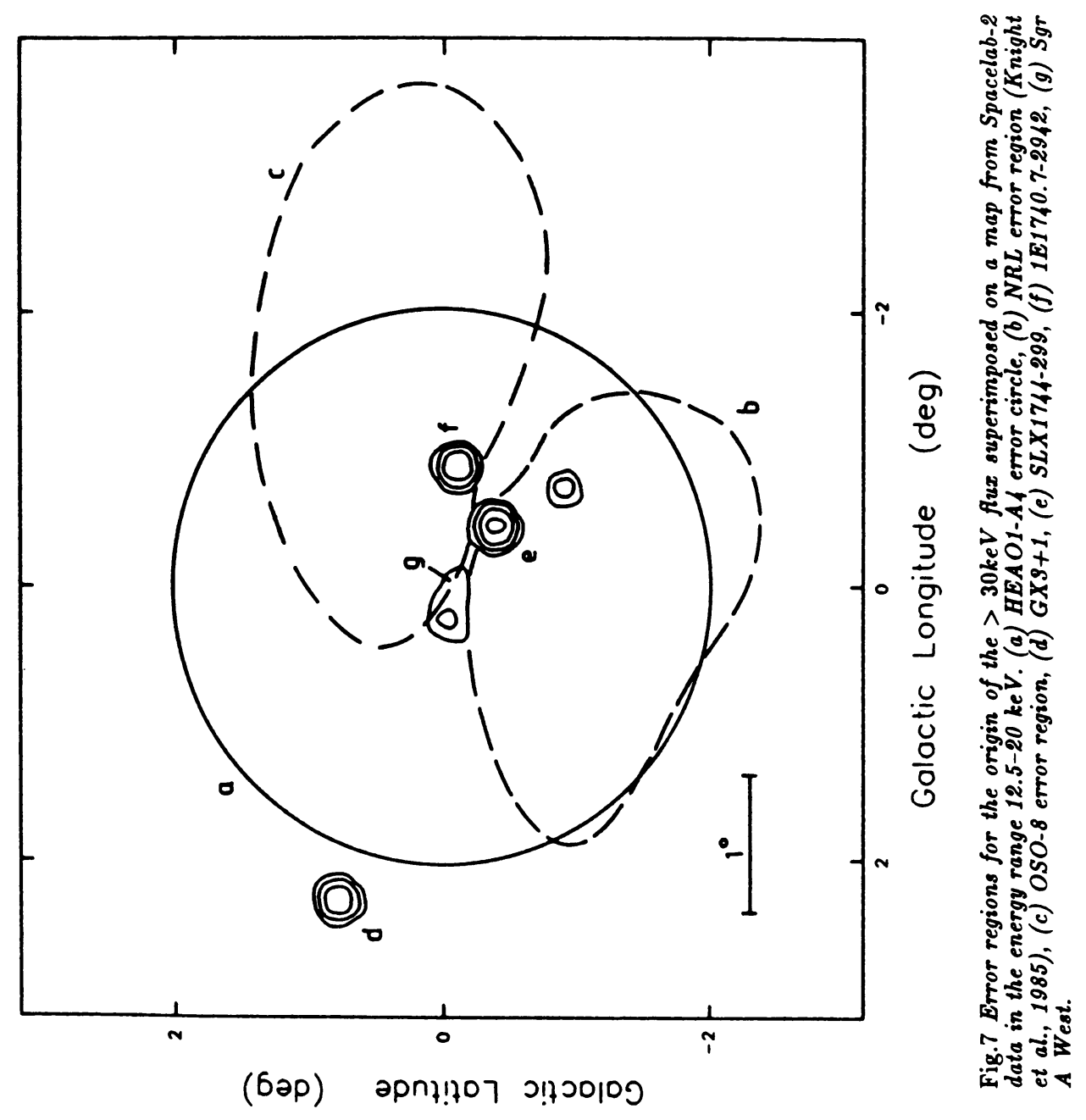


Spartan-1/Spacelab-2 observations than an extrapolation to higher energies of the Einstein results.

Thus a source positionally consistent with $\mathrm{Sgr} \mathrm{A}^{*}$ has been detected and is either variable (and hence compact) or more luminous at higher energies than expected on the basis of the Einstein observations. Even in the Spacelab-2 data its luminosity is only $6 \times 10^{35} \mathrm{erg} \mathrm{s}^{-1}$ (3-30 keV, allowing for low energy absorption) - much less than several other sources in the central $\sim 1^{\circ}$. For comparison with other Einstein observations the $0.5-4.5 \mathrm{keV}$ luminosity of $1.5 \times 10^{35} \mathrm{erg} \mathrm{s}^{-1}$ found by Watson et al. (1981) may be more relevant. On either basis, the nuclear emission is far weaker than that observed with the Einstein observatory from the nucleus of M31 (10 $38 \mathrm{erg} \mathrm{s}^{-1}$ Long and van Speybroeck, 1983), M33 (10 ${ }^{39} \mathrm{erg} \mathrm{s}^{-1}$ - Long et al., 1981; Markert and Rallis, 1983) or M100, M81 (10 ${ }^{40} \mathrm{erg} \mathrm{s}^{-1}$ - Pàlumbo et al., 1981; Fabbiano, 1988). Fabbiano and Trinchieri (1987) report and review data on nuclear x-ray emission from a number of spiral galaxies. Selection effects are, of course, such that except for nearby galaxies positive detections are only possible in those cases where the luminosity is high.

\section{INTERSTELLAR ABSORPTION}

The low energy cut-off of an $x$-ray spectrum is conventionally fitted to an absorption law based on a model for the composition of interstellar material such as that of Brown and Gould (1970) or of Morrison and MacCammon (1983) and the result expressed as the corresponding Hydrogen column density, $N_{H}$. Values found for the galactic centre sources and overall galactic centre $x$-ray emission have covered the range about $3 \times 10^{22}-3 \times 10^{23} \mathrm{~cm}^{-2}$. Some of this column may of course be local to the source, but the consistently high values found for sources in the region suggest that a major component is the interstellar material between the galactic centre region and the Sun. With column densities as high as this several effects come into play.

(a) The form of the low energy cut-off is dictated largely by the quantity of elements around Oxygen, rather than Hydrogen. If it is assumed that elements heavier than Helium have the same abundances relative to each other as used by Morrison and MacCammon (1984), then almost irrespective of the metallicity, $M$, we can characterize the absorption by the Carbon column density and place a limit $N_{C}<1.3 \times 10^{20} \mathrm{~cm}^{-2}$. The estimate is little affected if the heavy elements are bound in molecules or in grains.

(b) The total number of electrons in the material is such that Compton scattering of $\mathrm{x}$ - and gamma-rays is not negligible (Fabian, 1977; Skinner et al., 1987). For $N_{H}=10^{23} \mathrm{~cm}^{-2}, M=1$, the probability of scattering of $\mathrm{keV}$ x-rays is about $8 \%$. Provided $E_{\nu} \ll m c^{2}$ the energy change is of course small. This process must provide a small but perhaps significant component to the diffuse emission discussed above.

(c) If the fraction of the material in the form of grains is similar to that found in other directions, small angle scattering of low energy x-rays will be important. Because the scattering occurs much closer to the source than to the observer, the arrival directions will be smeared by only $\lesssim 1^{\prime}$, but more than $50 \%$ of the photons in the Einstein energy range may have been affected (Skinner et al., 1987).

If the effects of absorption, scattering by electrons and scattering by grains, which all have different signatures, can be separated out then $x$-rays will provide a means 
of estimating the amounts of Hydrogen, of heavy elements and of material within grains in the interstellar material.

\section{HARD X-RAYS (10-500 keV)}

There have been many detections of hard x-ray flux from the galactic centre region, but almost all with instruments having limited angular resolution, mostly $\geq 5^{\circ}$. In the HEAO-1 A4 catalogue (Levine et al., 1984) significant flux in the 13-180 keV range was reported from 6 sources within $5^{\circ}$ of $\operatorname{Sgr} \mathrm{A}$, so source confusion has been a major problem. A source at the position of Sgr A or of A1742-294 has usually been used in the fitting procedures used to estimate the flux from the separate components.

The hard $\mathrm{x}$-ray emission from the GCX region is highly variable. The HEAO-1 $A 4$ data (Matteson, 1982; Levine et al., 1984) showed a factor of two variation between March and September 1978 in the $80-180 \mathrm{keV}$ flux. This flux was attributed to A1742-294 for the purpose of the analysis, but the data only show that the source was within $\sim 2^{\circ}$ of the galactic centre. Even the average flux in the $80-180 \mathrm{keV}$ band, was larger than that from any other source in the galaxy except for the Crab Nebula and Cyg-X1. Assuming the 'A1742-294' flux originated at the distance of the galactic centre, and allowing for relative distances, then the intrinsic luminosity of the source at these energies was greater than that of any other source in the galaxy.

Earlier data were obtained in September and November 1977 with OSO-8 (Dennis et al., 1980) and from a NRL balloon flight (Knight et al., 1985). Each of these experiments operated in the range 20-200 keV. As shown in Fig.6, the spectra obtained were each (at least marginally) consistent with the range observed with $H E A O-1 \mathrm{~A}$ 4. Fig.6 also indicates the range of spectra which have been observed in the $200-500 \mathrm{keV}$ range. All of the instruments involved had angular resolution poorer than $12^{\circ}$ and it is likely that other sources and perhaps extended emission from positronium continuum are contributing. Nevertheless the relationship to the $H E A O-1 / O S O-8 / N R L$ spectra encourages one to think that at least part of the $>200 \mathrm{keV}$ flux may be related to that seen at lower energies.

The HEAO-1/OSO-8/NRL error regions obtained for the galactic centre emission were (again marginally) consistent with each other, with Sgr A, and with A1742294 (Fig.7). The 1985 Spacelab-2 observations make it seem most likely that the origin of the high energy flux is neither Sgr A nor A1742-294 but 1E1740.7-2942 which was by far the strongest source observed in the $6^{\circ} \times 6^{\circ}$ galactic centre field in the 20-32 $\mathrm{keV}$ range. It lies close to the edges of both the OSO-8 and NRL 95\% probability regions and within the HEAO-1 error circle. Radio and infrared maps show no particular features at the position of this unique object.

\section{CONCLUSIONS}

The central few parsecs of our galaxy seem to house a low-energy x-ray source, but not a particularly powerful one. It is surrounded by a patch of (apparently) diffuse emission of unknown origin. Somewhere in the vicinity lies a source of high-energy x-rays as powerful as any in the galaxy. We don't know where the high energy source is (although there are grounds for guessing that it may be $1 \mathrm{E} 1740.7-2942)$ or the nature of the object involved. 
The relatively low density of $x$-ray sources in other parts of the sky, together with the possibility of observing optical candidates, has enabled considerable progress to be made with modest angular resolutions. In the galactic centre there is a compact complex of sources and no possibility of complementary optical observations and so $x$-ray studies of the region have always been limited by imprecise knowledge of the directions from which the $x$-rays are coming.

This is a situation which should change radically in the future. We can look forward to $x$ - and gamma-ray imaging with a number of balloon experiments, with SIGMA, GRO and perhaps with GRASP and/or NAE and eventually, we hope, to sub-arcsecond observations with AXAF. There is even a proposal for placing grids, masks and a detector array on the 32 metre long CASES boom and to obtain subarcsecond images of the galactic centre with energies up to $100 \mathrm{keV}$. Perhaps in due course we may see the $x$-ray equivalents of the beautiful results being obtained with the VLA.

\section{REFERENCES}

Bhat, C.L., Kifune, T., and Wolfendale, A.W., 1985, Nature 318 267-269.

Branduardi, G., Ives, J.C., Sanford, P.W., Brinkman, A.C., Maraschi, L., 1976, Mon. Not. R. Astr. Soc. 175 47P-56P.

Brown, R.L., and Gould, R.J., 1970, Phys. Rev. D $12252-2256$.

Cruddace, R.G., Fritz, G., Shulman, S., Friedman, H., McKee, J., and Johnson, M., 1978, Ap. J. 222 L95-L98.

Davies, R.D., Walsh, D., Browne, I.W.A., Edwards, M.R., and Noble, R.G., 1976, Nature 261 476-478.

Dennis, B.R., Beall, J.H., Cutler, E.P., Crannell, C.J., Dolan, J.F., Frost, K.J., and Orwig, L.E., 1980, Ap. J. 236 L49-L53.

Ebisuzaki, T., Hanawa, T., and Sugimoto, D., 1984, Publ. Astron. Soc. Japan 36 551566.

Eyles, C.J., Skinner, G.K., Willmore, A.P., and Rosenberg, F.D., 1975, Nature 257 291-293.

Fabian, A.C., 1977, Nature 269 672-673.

Fabbiano, G., 1988, Ap. J. 325 544-562.

Fabbiano, G., and Trinchieri, G., 1987, Ap. J. 315 46-67.

Hayakawa, S., 1981, Space Sci. Rev. 29 221-290.

Inoue, H., Koyama, K., Makishima, K., Matsuoka, M., Murakami, T., Oda, M., Ogawara, Y., Ohashi, T., Shibazaki, N., Tanaka, Y., Hayakawa, S., Kunieda, K., Makino, F., Masai, K., Nagase, F., Tawara, Y., Miyamoto, S., Tsunemi, T., and Yamashita, Y., 1981, Ap. J. 250 L71-L75.

Hertz, P., and Grindlay, J.E., 1984, Ap. J. 278 137-149.

Jernigan, J.G., Apparao, K.M.V., Bradt, H.V., Doxsey, R.E., Dower, R.G., and McClintock, J.E., 1978, Nature, 272 701-704.

Kawai, N., Fenimore, E.E., Middleditch, J., Cruddace, R.G., Fritz, G.G., Snyder, W.A., and

Ulmer, M.P., 1988, Ap. J. 330 130-141.

Kellogg, E., Gurski, H., Murray, S., Tananbaum, H., and Giacconi, R., 1971, Ap. J. , 169 L99-L103. 
Knight, F.K., Johnson, W.N., III, Kurfess, J.D., and Strickman, M.S., 1985, Ap. J. 290 557-567.

Koyama, K., Makishima, K., Tanaka, Y., and Tsunema, H., 1986, Publ. Astr. Soc. Jpn. 38121.

Leventhal, M., MacCallum, C.J., and Stang, P.D., 1978, Ap. J. 225 L11-L14.

Levine, A.M., Lang, F.L., Lewin, W.H.G., Primini, F.A., Dobson, C.A., Doty, J.P., Hoffman, J.A., Matteson, J.L., Baity, W.A., Gruber, D.E., Knight, F.K., Nolan, P.L., Pelling, R.M., Rothschild, R.E., and Peterson, L.E., 1984, Ap. J. 54 581-617.

Lewin, W.G.H., Hoffman, J.A., Doty, J., Hearn, D.R., Clark, G.W., Jernigan, J.G., Li, F.K., McClintock, J.E., and Richardson, J., 1976, Mon. Not. R. Astr. Soc. 177 83P-92P.

Lewin, W.G.H., and Joss, P.C., 1981, Space Sci. Rev. 28 3-87.

London, R.A., Taam, R.E., and Howard, W.M., 1984, Ap. J. 287 L27.

Long, K.S., D'Odorico, S., Charles, P.A., and Dopita, M.A., 1981, Ap. J. 246 L61-L64.

Long, K.S., and Van Speybroeck, L.P., 1983, Accretion-driven stellar x-ray sources eds. W.H.G. Lewin and E.P.J. Van den Heuvel, C.U.P., Cambs., 117-146.

Markert, T.H., and Rallis, A.D., 1983, Ap. J. 275 571-577.

Matteson, J.L., 1982, The Galactic Center Eds. G.R. Riegler and R.D. Blandford, AIP, New York, 109-122.

McClintock, J.E., and Rappaport, S.A., 1985, Cataclysmic Variables and Low-Mass $X$-ray Binaries Eds. D.Q. Lamb and J . Patterson, Reidel, Dordrecht, 61-77.

Morrison, R., and MacCammon, D., 1983, Ap. J. 270 119-122.

Murdin, P., Allen, D.A., Morton, D.C., Whelan, J.A.J, and Thomas, R.M., 1980, Mon. Not. R. Astr. Soc. 192 709-717.

Palumbo, G.G.C., Maccacaro, T., Panagia, N., Vettolani, G., and Zamorani, G., 1981, Space Sci. Rev. 30 143-147.

Proctor, R.J., Skinner, G.K., and Willmore, A.P., 1978, Mon. Not. R. Astr. Soc. 185 745-754.

Skinner, G.K., 1979, Proc. Roy. Soc. Lond. A 366 245-255.

Skinner, G.K., Willmore, A.P., Eyles, C.J., Bertram, D., Church, M.J., Harper, P.K.S., Herring, J.R.H., Peden, J.C.M., Pollock, A.M.T., Ponman, T.J., 1987, Nature 330 544-547.

Tanaka, Y., 1987, The Origin and Evolution of Neutron Stars, eds. D.J. Helfand and J.H.Huang, Reidel, Dordrecht, 161-172.

Warwick, R.S., Turner, M.J.L., Watson, M.G., and Willingale, R., 1985, Nature 317 218-221.

Warwick, R.S., Norton, A.J., Turner, M.J.L., Watson, M.G., and Willingale, R., 1988, Mon. Not. R. Astr. Soc. 232 551-564.

Watson, M.G., Willingale, R., Grindlay, J.E., and Hertz, P., 1981, Ap. J. 250 142-154.

Wilson, A.M., Carpenter, G.F., Eyles, C.J., Skinner, G.K., and Willmore, A.P., 1977, Ap. J. 215 L111-L115.

Worrall, D.M., and Marshall, F.E., 1983, Ap. J. 267 691-697.

Yusef-Zadeh, F., and Bally, J., 1987, Nature 330 455-458. 